lactic administration of antibiotics. ${ }^{3}$ Nevertheless, the most convincing piece of evidence is still lacking-namely, the reproduction of the disease in animals by infection with an appropriate strain of streptococcus. For this reason the recent claim by workers at the Pasteur Institute in Paris ${ }^{4}$ to have induced arthritis and carditis in mice infected with streptococci is of unusual interest.

Previous attempts to produce similar lesions in mice have failed because most strains of streptococci contain a proportion of highly virulent individuals (BM organisms) which kill the animals before lesions have time to develop. Strains deficient in these BM variants (so-called vm organisms) also fail to induce lesions because they are rapidly eliminated by the macrophages in the host. P. Cayeux and his colleagues ${ }^{4}$ report having overcome this drawback by employing strains of streptococci relatively poor in BM organisms and by suppressing the activity of the host macrophages by treating the animals with a rabbit antimouse-macrophage antiserum one hour before injection of the organisms. Of 300 mice treated in this way 6\% developed an arthritis of the wrists and ankles and about $50 \%$ enlargement of the heart. On histological examination the affected synovia were infiltrated with lymphocytes, plasma cells, and disintegrated polymorphs, and the affected hearts showed foci of necrosis and scattered areas of histiocytic infiltration. Both by microscopy as well as by bacteriological culture organisms were readily found in all the lesions.

Before these findings can be accepted as throwing light on the relationship between streptococcal infection and rheumatic fever the important differences between the human and mouse diseases must not be overlooked. In man there is a highly characteristic quiescent interval of two to three weeks between the primary streptococcal infection and the clinical appearance of joint or cardiac symptoms. In the mouse, on the other hand, arthritis was found to occur occasionally as early as the second day. In man, when special steps are taken to avoid contamination, the lesions are invariably sterile ${ }^{5}$; in the mouse they are obviously the result of local invasion by the living organisms. Rheumatic fever, even in severe epidemics of streptococcal infection, rarely affects more than $3 \%$ of the patients; in the infected mice the heart was affected in $50 \%$ of cases. Finally, the histological nature of the experimental lesions bore no resemblance either in the joints or in the heart to those of the rheumatic person. Thus readers may well conclude that the mechanism relating streptococci to rheumatic fever has yet to be produced in the experimental animal.

\section{Carcinoma of Hypopharynx and Cervical Oesophagus}

Carcinoma of the hypopharynx (post-cricoid) and the cervical oesophagus is fortunately uncommon. When it presents the primary lesion is usually extensive and often accompanied by metastatic nodes. Treatment, whether by radiotherapy or surgery, ${ }^{1}$ has been generally unsatisfactory and $50 \%$ of patients have died after a distressing illness within the first year of treatment. Because fibrous strictures, oedema, and local recurrence commonly occurred after irradiation, the predominant symptom of dysphagia was not relieved, and, since tracheal or laryngeal obstruction supervened, the patient was left to die with a tracheostomy and permanent feeding through an oesophageal catheter or a gastrostomy. Not only was the rate of long-term cure poor but palliation was also unsatisfactory.

Surgeons have argued that pharyngolaryngectomy combined with unilateral or bilateral neck dissection in continuity, though carrying a mortality rate of $3 \%$ to $12 \%,,^{2}$ offered a better chance of survival. Pharyngeal reconstruction by skin flaps took a minimum of six weeks in non-irradiated cases and much longer in those previously irradiated-so long, in fact, that many reconstructions were never completed because of local recurrences and metastases. To overcome this delay in the restoration of swallowing immediate methods of reconstruction were devised using fascia or skin grafts round moulds, pieces of trachea, vascular homografts, and plastic prostheses, but all were bedevilled by salivary fistulas and strictures. $^{3-6}$ Further, of those patients suitable for surgery less than $20 \%$ survived five years, only a third more than two years, ${ }^{7}$ and many died unrelieved of distress. Not surprisingly, the disease has been regarded as virtually incurable, and a safe, immediate method of restoring swallowing that will serve for what remains of the patient's life has been sought as a palliative.

Since pedicle grafts of stomach, jejunum, or colon had previously been used successfully for immediate oesophageal replacement, the method was extended to the pharynx. ${ }^{1-11}$ Subcutaneous and retrosternal routes to by-pass the gullet were first used, but, as recurrences in the oesophageal stump were common, the initial excision was extended to include the whole oesophagus and the tunnel so created was used for routing the gastric ${ }^{12-14}$ and colonic transplants. Free transplants of jejunum and colon, revascularized by anastomosis of the mesenteric vessels to convenient vessels in the neck, have also been used. ${ }^{611}$

However, all transplantations are formidable procedures and carry a mortality of $30 \%-50 \%$. That is a high price to pay for palliation when it is known that two-thirds of the patients will die within two years, no matter what the extent of the excision. Recently a non-slip Polyvinyl tube has been made to replace the pharynx after a wide cervical excision. ${ }^{15}$ The operative mortality from the procedure is low and the palliative results good, but after it patients must mince all the meat they eat. Occasionally the anterior parts of the larynx and trachea can be preserved to form a new anterior wall for the pharynx, the posterior wall being fashioned by a skin graft on to the prevertebral muscles. ${ }^{11}{ }^{16-18}$ A major sequel to pharyngolaryngectomy is a loss of voice, but after replacement with a colon graft several patients have developed a useful " oesophageal " voice. ${ }^{19}$

All these newer techniques have failed to offer a satisfactory outcome for most patients, and it is hoped that more modern radiotherapy techniques and cytotoxic drugs may offer better prospects.

\footnotetext{
1 Fairman, H. D., Hadley, S. K. J., and John, H. T., Brit. F. Surg., 1964, 51, 663.

2 Ranger, D., Proc. roy. Soc. Med., 1964, 57, 1099.

3 Shaw, H. J., ibid., 1964, 57, 1108 .

- Rob, C. G., and Bateman, G. H., Brit. F. Surg., 1949, 37, 202.

s Negus, v. E., Proc. roy. Soc. Med., 1950, 43, 168.

Harrison, D. F. N., ibid., 1964, 57, 1104.

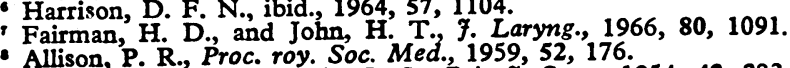

Goligher J. C., and Robin I. G. Brit. 7. Surg., 1954, 42, 283.

10 Gobbs, C. R., and Mullard, K.,

11 Simpson, J. F., ibid, 1966, 80, 1077.

12 Le Quesne, L. P., Proc. roy. Soc. Med., 1964, 57, 1103.

13 Ong, G. B., Brit. F. Surg., 1964, 51, 53.

14 Stuart, D. W., T. C., ibid., 1960, 48, 193.

16 Stuart, D. W., f. Laryng., 1966, 80,

17 Som, M. L., Arch. Otolaryng., 1956, 63, 474.

13 Simpson, J.'F., 尹. Laryng., 1960, 74, 300.

10 Lall, M., and Evison, G., ibid., 1966, 80, 1208.
} 